\title{
Il quadraturismo in Pallazzo Pitti da Cosimo II a Cosimo III de' Medici ${ }^{1}$
}

\section{The quadraturism on the Pallazzo Pitti from Cosimo II to Cosimo III of Medici}

FaUZIA FARNetI

Docente da Facoltà di Architettura, Dipartimento di restauro e conservazione dei beni architettonici, Università degli studi di Firenze via Micheli 8 Firenze; e-mail: farneti@unifi.it

\begin{abstract}
RESUMO Nei primi decenni del Seicento la pittura decorativa a Firenze risulta ancora legata all'ornamentazione tradizionale tardomanierista attuata nei modi di Alessandro Allori o di Bernardino Barbatelli detto il Poccetti. L'interesse per le novità e per l'aggiornamento dell'ambiente artistico fiorentino portarono il granduca Ferdinando II a chiamare a Firenze tra il 1636 ed il 1637 Pietro da Cortona, Angelo Michele Colonna e Agostino Mitelli. I due bolognesi completarono il ciclo pittorico celebrativo del governo di Ferdinando cui aveva dato inizio Giovanni da San Giovanni, con la decorazione delle tre sale di rappresentanza del quartiere estivo di palazzo Pitti realizzata tra il 1637 ed il 1641. L'intervento, condotto secondo il più moderno linguaggio barocco che vede la perfetta integrazione dell'illusionismo architettonico, che
\end{abstract}

1 Artigo recebido em Março de 2008. Autora convidada. 
supera i limiti dello spazio reale, con le scene figurative, verrà a costituire nell'ambiente fiorentino un ineludibile modello di riferimento nella decorazione d'interni, soluzioni di grande modernità su cui si formerà J acopo Chiavistelli e i giovani della sua scuola. Anche Giovan Carlo, fratello del granduca, nel 1637 diede inizio ad una serie di trasformazioni che si protrassero per oltre un ventennio, trasformando gli ambienti a lui assegnati in Pitti in veri e propri luoghi di delizie, decorati dagli artisti più significativi del momento quali ad esempio Angelo Michele Colonna, Agostino Mitelli, Pietro da Cortona, J acopo Chiavistelli. Fu quest'ultimo frescante che con i suoi 'scolari', fin dagli anni Cinquanta fu attivo in palazzo Pitti, decorand o a quadratura gli ambienti dei quartieri dei membri della famiglia granducale, ambienti che in gran parte sono andati perduti in quanto interessati dalle ristrutturazioni lorenesi e sabaude. Con i lavori commissionati dal gran principe Ferdinando si chiude in palazzo Pitti la grande stagione del quadraturismo barocco fiorentino.

Parola chiave quadraturismo; barocco; Firenze

ABSTRACT In the early decades of the 17 th century decorative painting in Florence is still tied to traditional ornamentation late-mannerist by Alessandro Allori or Bernardino Barbatelli who is called Poccetti. Between 1636 and 1637, the Grand Duke Ferdinando the 2nd called to Florence Pietro da Cortona, Angelo Michele Colonna e Agostino Mitelli to upgrade the artistic environment. Between 1637 and 1641, with the decoration of three representation rooms, the two bolognese completed the painting cycle who celebrating the Ferdinando's Government, started by Giovanni da San Giovanni in the summer residence of Palazzo Pitti. The intervention, according most modern baroque language, who sees the perfect integration of architectural illusionism and exceeds the limitations of real space with the figurative, will be in Florentine environment inescapable reference model in interior decorating, great modern solutions on which will be formed J acopo Chiavistelli and his school. Even Giovan Carlo, brother of G rand Duke, in 1637 began a series of transformations that lasted for over twenty years, turning the rooms assigned to him in Pitti in places of delights, decorated by the most significant artists of the moment such as Angelo Michele Colonna, Mitelli Agostino, Pietro da Cortona, J acopo Chiavistelli. It was the latter frescante, active in Pitti Palace since 1650 ,that with his' pupils' decorated in squaring the neighborhoods of members of the grand ducal family, environments largely lost because of the restructuring of Lorena and Savoy. With the work commissioned by Prince Ferdinando in Pitti Palace they closed the great season of squaring in the Florentine baroque.

Keywords quadraturism; baroque; Florence 
RESUMO Nos primeiros decênios do Seiscentos a pintura decorativa em Florença resulta ainda ligada à ornamentação tradicional tardo maneirista operado nos moldes de Alessandro Allori ou nos de Bernadino Barbatelli, chamado de Poccetti. Os interesses pela novidade e pela modernização do ambiente artístico florentino levaram ao grão duque Ferdinando II a chamar para Florença, entre 1636 e 1637, Pietro da Cortona, Angelo Michele Colonna e Agostino Mitelli. Os dois bolonheses completaram o ciclo pictórico comemorativo do governo de Ferdinando, que tinha sido iniciado por Giovanni da San Giovanni na decoração das três salas de representação do apartamento de verão do palácio Pitti, realizada entre 1637 e 1641. A intervenção conduzida segundo a mais moderna linguagem barroca, a partir de uma perfeita integração do ilusionismo arquitetônico supera os limites do espaço real com cenas figurativas. Deste modo, seria constituído no ambiente florentino um ineludível modelo de referência na decoração dos interiores, soluções de grande modernidade nas quais se formará J acopo Chiavistelli e os jovens da sua escola. Também Giovan Carlo, irmão do grão duque, em 1637 dá início a uma série de transformações que se prolongaram por mais de vinte anos, transformando os ambientes assinados por ele no palácio Pitti em verdadeiros e próprios lugares de deleites, decorados por artistas mais significativos do momento, dos quais, por exemplo, Angelo Michele Colonna, Agostino Mitelli, Pietro da Cortona, J acopo Chiavistelli. Foi este ultimo pintor de afrescos quem, com os seus "alunos", trabalhou no Palazzo Pitti desde os anos Cinquenta, decorando os ambientes dos apartamentos dos membros da familia dos grão duques com quadraturas. Grande parte desses ambientes desapareceu, pois foram reformados durante o governo dos Lorenas e dos Sabaudas. Com os trabalhos comissionados pelo grão duque príncipe Ferdinando se fecha no palácio Pitti o período do quadraturismo barroco florentino.

Palavras-chave quadraturismo; barroco; Florença

Nei primi decenni del Seicento la pittura decorativa a Firenze risulta ancora legata all'ornamentazione tradizionale tardomanierista attuata nei modi di Alessandro Allori o di Bernardino Barbatelli detto il Poccetti. Si tratta di pittura a grottesca 0 ad imitazione di finti stucchi a forma di ghirlande 0 di erme, che occupa spesso il soffitto circondando le decorazioni a scene storiche e di paesaggio, imitando le cornici sino a creare quadri riportati. La parte alta delle pareti è generalmente percorsa da un fregio realizzato nella stessa modalità, mentre il resto di esse viene ricoperto di stoffa: protagonista di questo genere decorativo fu Michelangelo Cinganelli, allievo del Poccetti, molto attivo nella decorazione delle sale di palazzo Pitti e, fra 
queste, si segnala la cappella del pianterreno nell'andito di passaggio tra il salone di Giovanni da San Giovanni e la prima sala dell'Udienza, e quella delle Reliquie per l'arciduchessa Maria Maddalena d'Austria. Marco Chiarini suppone che quest'ultimo intervento del Cinganelli, artista preferito da Maria Maddalena, sia stato realizzato nell'estate del 1628 mentre Elisa Acanfora lo anticipa al $1622 .^{2}$

Interessante sottolineare che sino a questo momento, ai primi decenni del Seicento, la prospettiva veniva studiata per essere usata solo nella rappresentazione di scene non ornamentali e soprattutto nella scenografia, e a questo scopo veniva insegnata da Giulio Parigi, che teneva «in casa sua una scuola, o vogliamo dire accademia» ${ }^{3}$ dove si concentrarono le ricerche prospettiche. Pittore, ingegnere ed architetto, a partire dal 1621, secondo la volontà espressa da Cosimo II, il Parigi ampliò e ristrutturò palazzo Pitti e, non a caso si è occupato di allestimenti scenografici; ${ }^{4}$ come riferisce il Baldinucci, in quel periodo «non si messe poi mano a fabbrica o a nobile apparato in Firenze, che non fusse inventato, maneggiato, perfezionato da lui». ${ }^{5} \mathrm{Fu}$ senza dubbio una figura poliedrica che rivestì un ruolo significativo nella definizione degli orientamenti e del gusto della decorazione murale di questo periodo. ${ }^{6}$

In seguito un altro pittore non architetto, il versatile Baccio del Bianco, ${ }^{7}$ come ci informa sempre il Baldinucci, aprì una pubblica scuola di prospettiva e di civile e militare architettura, ed ebbe tra i suoi allievi J acopo Chiavistelli, Agnolo Gori e Andrea Ciseri, tra i più attivi decoratori e pittori prospettici del barocco fiorentino. ${ }^{8}$ Anche Baccio del Bianco si adoperò nella scenografia teatrale; nel 1637 ad esempio, quando assieme ad Alfonso Parigi allesti la macchina teatrale e le prospettive per la commedia Le nozze degli dei dell'abate Coppola, in occasione delle nozze di Ferdinando con Vittoria della Rovere. Le decorazioni delle sale di rappresentanza nel quartiere terreno di palazzo Pitti, che oggi fanno parte del museo degli Argenti, furono affidate

2 ACANFORA, E. La decorazione delle loggette. Gli anni della reggenza di Maria Maddalena d'Austria e oltre. In: Palazzo Pitti. Firenze 2000, p. 57-62 (p. 62 nota 21).

3 BALDINUCCI, F. Notizie de Professori del disegno da Cimabue in qua, 1681-1728. ed. a cura di F. Ranalli, Firenze 1845-47, IV, 1846, p. 141, 145, 198.

4 Filippo Baldinucci infatti riferisce che il Parigi «insegnava le macchine, prospettiva, architettura civile e militare»: BALDINUCCI, F. (1681-1728), ed. 1845-1847, IV, 1846, p. 663-664; BALDINUCCI, F.S. Vite di artisti dei secoli XVII e XVIII, (1725-1730c.), ed. a cura di A. Matteoli, Roma 1975, p. 276.

5 BALDINUCCI, F. (1681-1728), ed. 1845-1847, IV, 1846, p. 123.

6 Sull'importanza del Parigi cf. MANNINI, M.P. Decorazioni fiorentine del Seicento tra commedia d'arte e melodramma. In: «Paragone», XLV, nuova serie, nn. 44-46, 1994, p. 220-230 (p. 225-226); ACANFORA, E. Pittura murale a Firenze dalla reggenza a Ferdinando II de' Medici. In: Pietro da Cortona, a cura di CH. L. Frommel, S. Schütze, atti del convegno (Roma-Firenze 12-15 novembre 1997). Milano, 1998, p. 145-162; ACANFORA, E. La pittura ad affresco fino a Giovanni da San Giovanni. In: Storia delle arti in Toscana. II Seicento, a cura di M. Gregori, Firenze 2001, P. 45-60 (p. 49); BUCCHERI, A. II ruolo della scenografia da Bernardo Buontalenti a Giulio Parigi. In: Storia delle arti in Toscana. II Seicento, a cura di M. Gregori. Firenze, 2001, p. 21-28.

7 Baccio del Bianco fu attivo anche per Don Lorenzo, realizzando nella villa di Castello «cose belle e curiose». Alcuni dei suoi celebri disegni sono conservati al Gabinetto Disegni e Stampe degli Uffizi e presso la Biblioteca Nazionale Centrale di Firenze.

8 ARCANGELI, A. Baccio del Bianco. In: II Seicento fiorentino. Arte a Firenze da Ferdinando I a Cosimo III, catalogo della mostra (Firenze 1986-1987), 3 voll. Firenze, 1986, Biografie, p. 76-78 (p. 76). 
al celebre Giovanni da San Giovanni, ${ }^{9}$ che Giulio Parigi, suo maestro «nella prospettiva e architettura», ${ }^{10}$ alcuni anni prima aveva consig liato alla granduchessa Maria Maddalena d'Austria per le pitture murali della fabbrica di Poggio Imperiale in quanto lo «stimava il migliore di quanti a quel tempo dipingessero a fresco $» .11^{11}$

Giovanni diede inizio ai lavori nell'anticamera della sala dell'Udienza nel maggio del 1635, progettando l'ingannevole apparato architettonico di grande monumentalità coinvolgente le pareti e il soffitto in cui mostra la comprensione e la sua adesione alle novità decorative romane, ai modelli più aggiornati sul gusto barocco. ${ }^{12}$ Suscita meraviglia quella «forza di scuri e di lumi», quel contrasto tra «gli scuri gagliardi dati dal pittore ne' sottosquadri, ed il lume » che ha una funzione primaria nella definizione dell'illusionismo architettonico come nella definizione plastica delle figure. II portico che si dispiega sulle pareti del salone con una soluzione da lui già adottata nella villa del Pozzino, mostra una evidente analogia con il portico terreno del salone del primo piano di palazzo Lancellotti a Roma, che possiamo definire una grandiosa illusione, realizzato nel 1617 da Agostino Tassi, trovando precedenti nella peruzziana sala delle Prospettive. ${ }^{13}$ Sulle illusionistiche arcate, che coincidono con quelle reali del cortile retrostante, in quella commistione di realtà e finzione che informa tutta la progettazione, si imposta la volta che dilata ulteriormente le dimensioni della sala. Questo costrutto di grande efficacia illusionistica, rappresentato in rapporto allo spettatore posto al centro dell'ambiente, rimanda ad apparati romani e potrebbe essere stato progettato dal Mannozzi con l'aiuto di Baccio del Bianco, suo collaboratore nella sala di Ebe della villa di Mezzomonte di Giovan Carlo de' Medici secondo quanto riferisce il Baldinucci, e di Bartolomeo Neri. Sempre a detta del Baldinucci collaborò alle pitture del salone granducale il giovanissimo Volterrano che avrebbe realizzato «in una cantonata sopra un cimiero, al-

9 Giovanni da San Giovanni fu uno degli artisti preferiti anche dal principe Don Lorenzo, colto collezionista, al quale commissionò la bellissima serie dei "capricci" a intonaco su paniera di stuoia, oggi agli Uffizi, e l'ironica tela con La prima notte di nozze per la villa di Castello, regalato dal principe al nipote Ferdinando II in occasione del suo matrimonio con Vittoria della Rovere; oggi si trova in palazzo Pitti; cfr. BOREA, E. La quadreria di Don Lorenzo de' Medici. Firenze, 1977, p. 13-26, p. 47-50, 146-147.

10 BALDINUCCI, F. (1681-1728), ed. 1845-47, IV, 1846, p. 141

11 BALDINUCCI, F. (1681-1728), ed. 1845-1847, IV, 1846, p. 234-235. Fu autore dell'affresco sulla facciata «incontro alla Porta a S. Piero Gattolini», piazza della Calza, commissionato nel 1616 da Cosimo Il e pagatogli nel 1622, essendo intervenuto come intermediario Giulio Parigi. I documenti sono stati resi noti da Elisa Acanfora in ACANFORA, E. La villa di Poggio Imperiale. In: Fasto di corte, a cura di M. Gregori, vol. I, Firenze 2005, p. 143-156 (p. 145 nota 72). Giovanni da San Giovanni rifiutò l'incarico per la dimora di Maria Maddalena in quanto impegnato a Roma nella chiesa dei Santi Quattro Coronati; comunque con lei ebbe insanati motivi di contrasto.

12 Prima di collaborare con Baccio del Bianco nella villa di Mezzomonte di Giovan Carlo de' Medici nel 1634, Giovann era stato a Roma dove aveva lavorato per i Bentivoglio con il Guercino che, per le architetture dipinte, si avvaleva di Agostino Tassi.

13 Purtroppo è andata perduta la decorazione pittorica realizzata dal Poccetti in palazzo Acciaioli e per i quali si rimanda a LEONCINI, G. Una "Vita di J acopo Chiavistelli pittore di figure et eccellente nell'architettura a fresco. In: «Rivista d'arte», XXXVII, serie quarta, 1984, I, p. 269-346 (p. 274 e nota 12); ACANFORA, E. La pittura ad affresco fino a Giovanni da San Giovanni. In: Storia delle arti in Toscana. II Seicento. Firenze, 2001, p. 45-60 (p. 46). 
cuni spennacchi», ${ }^{14}$ che Malcom Campbell identifica nelle Storie di Paride raffigurate nei tondi in finto oro.

La soluzione compositiva adottata da Giovanni da San Giovanni sul soffitto dell'anticamera della sala dell'Udienza a palazzo Pitti, ${ }^{15}$ in cui si celebra I'unione matrimoniale di Ferdinando de' Medici con Vittoria della Rovere, ${ }^{16}$ pur di soggetto diverso, può essere messa in relazione con le scenografie del 1608 di Giulio Parigi. II motivo dello sfondato centrale delimitato da una cornice balaustrata la cui continuità è interrotta dalle nuvole che creano un'ombra portata sulle cornici architettoniche, rimanda al Rinaldo e Armida realizzato in palazzo Patrizi oggi Costaguti negli anni Venti dal Guercino in collaborazione con Agostino Tassi, che fu a Firenze alla corte di Ferdinando ed entrò in contatto con Giulio Parigi. ${ }^{17}$ Mina Gregori ipotizza un rapporto diretto del Mannozzi con Agostino Tassi nella definizione della cornice del Perseo in palazzo Rospigliosi, già Bentivoglio, a Roma. ${ }^{18}$

Baldinucci mette in evidenza le capacità di Giovanni da San Giovanni nel realizzare lo sfondato centrale del soffitto, la «veduta di sotto in su», vedendo in lui l'iniziatore a Firenze di inediti effetti di superamento dello spazio reale, vicini alla spazialità barocca. L'apparato decorativo della sala rimase incompiuto in quanto Giovanni, mentre attendeva alla decorazione del salone granducale, era molto attivo anche nel palazzo di Alessandro Pucci, e la morte lo colse prematuramente il 9 dicembre 1636.

La soluzione da lui adottata nell'anticamera della sala dell'U dienza, prima dell'arrivo di Pietro da Cortona e di Angelo Michele Colonna e Agostino Mitelli costituisce una novità nel panorama fiorentino, da vedere in rapporto al motivo architettonico messa a punto da Alessandro Allori nella loggetta del primo piano del palazzo prospettante il cortile dei Paggi, una straordinaria anticipazione dell'illusionismo architettonico barocco in palazzo Pitti.

La sala di Giovanni da San Giovanni di Pitti può essere un significativo precedente del completamento pittorico del cortile della villa La Pietraia ${ }^{19}$ commissionata da Don Lorenzo, quartogenito di Ferdinando I e Cristina

14 BALDINUCCI, F. (1681-1728), ed. 1845-1847, IV, 1846, p. 252-253; V, 1847, p. 145; CAMPBELL, M. The original Program of the Salone di Giovanni da San Giovanni. In: «Antichità Viva», XV, 1976, 4, p. 5-27 (p. 6 fig. 6).

15 L'ampliamento del palazzo con la definizione planimetrica del quartiere terreno nell'ala sinistra fu eseguito da Giulio e Alfonso Parigi a partire dal 1620, continuando il modello del nucleo originario.

16 Le vicende dell'impresa sono descritte da Filippo Baldinucci e chiarite ulteriormente dal Giglioli e da Campbell; GIGLIOLI, E.H. Giovanni da San Giovanni (Giovanni Mannozzi 1592-1636). Studi e ricerche. Firenze 1949; CAMPBELL, M. Medici Patronage and the Baroque. A Reappraisal. In: «The Art Bulletin», XLVIII, 1966, 2, p. 132-146; CAMPBELL, M. The original Program of the Salone di Giovanni da San Giovanni. In: «Antichità Viva», XV, 1976, 4, p. 5-27. Per i disegni si rimanda ai testi di CAMPBELL, M. The original Program of the Salone di Giovanni da San Giovanni. In: «Antichità Viva», XV, 1976, 4, p. 9, fig. 11 che ha riconosciuto anche la dipendenza da modelli di Giulio Parigi; DELLA MONICA, I. In: Giovanni da San Giovanni. Disegni, a cura di I. Della Monica, catalogo della mostra (San Giovanni Valdarno). Bologna, 1994, p. 88, figura a p. 89, p. 92, fig. a p. 93.

17 PASSERI, G.B. Vite di Pittori, Scultori, Architetti, Roma 1779. La formazione scientifica del Tassi, definito da Luigi Lanzi «pittore eccellente, ma uomo malvagio», che lasciò Firenze nel 1610, fu influenzata da Ludovico Cigoli, autore di Prospettiva pratica, completato nel 1612 ma mai dato alle stampe.

18 GREGORI, M. Arte fiorentina tra 'maniera' e 'barocco'. In: «Paragone», XV, 1964, 169, p. 11-23 (p. 18, 22, nota 24, Tav. 24).

19 Fino a questo momento erano dipinte solo le due facciate della villa. 
di Lorena, a Baldassarre Franceschini, detto il Volterrano, che diede inizio all'impresa alla fine del 1636. II principe, in sintonia con il nipote Giovan Carlo, dimostrandosi all'avanguardia nelle scelte artistiche, in un primo momento aveva deciso di dipingere le logge «non d'altro, che di prospettive, parendogli che un tal ornamento fosse per far comparire quel luogo di gran lunga più spazioso»ma, dissuaso da Lodovico Incontri fece rappresentare «alcune delle più gloriose azioni de' principi di quella sua serenissima casa» sui lati lunghi del cortile, commissionandole al Volterrano. ${ }^{20}$ Le quattro scene maggiori, relative alle gesta di Cosimo I, Ferdinando I, e ai principi ereditari Francesco (poi Francesco I) e Cosimo (poi Cosimo II) sono introdotte da un ingannevole apparato «nel mezzo del quale finse una fontana d'acqua, che esce da certe maschere ed arpie, col vaso che la riceve, e sopra la fontana l'arme di casa Medici», ${ }^{21}$ che cela il pianerottolo della illusionistica scala ellittica a due rampe che si dispiega sui due lati della fontana, scolpita sull'esempio di quelle poste sotto alcune finestre dell'appartamento degli Argenti a Pitti. ${ }^{22}$ Questo virtuale basamento alle scene figurative soprastanti, che viene a far parte dello spazio reale, crea un effetto di dilatazione delle scene oltre il limite della parete. Nelle testate delle logge il Volterrano ricorre ad un espediente diverso, rappresentando i personaggi di straordinaria naturalezza sullo sfondo delle due rampe della illusionistica scala posta lateralmente alla porta, memore dell'inganno prospettico adottato dal Colonna e dal Mitelli nella prima sala del quartiere di rappresentanza di palazzo Pitti, realizzato fra il 1637 ed il 1639. Il costrutto dell'inganno, rappresentato a fingere il marmo, mostra un ricco repertorio decorativo costituito da putti, festoni, mascheroni, bassorilievi con satiri e ninfe.

L'interesse per le novità e per l'aggiomamento dell'ambiente artistico fiorentino portarono Ferdinando II a chiamare a Firenze tra il 1636 ed il 1637 Pietro da Cortona, Angelo Michele Colonna e Agostino Mitelli. ${ }^{23}$ I due bolognesi completarono il ciclo pittorico celebrativo del governo di Ferdinando Il cui aveva dato inizio Giovanni da San Giovanni, con la decorazione delle tre sale di rappresentanza del quartiere estivo di palazzo Pitti realizzata il 1637 ed il 1641. L'intervento, condotto secondo il più moderno linguaggio barocco che vede la perfetta integrazione dell'illusionismo architettonico, che supera i limiti dello spazio reale, con le scene figurative, verrà a costituire nell'ambiente fiorentino un ineludibile modello di riferimento nella decorazione

20 Per approfondimenti si rimanda a SPINELLI, R. Gli affreschi di Baldassarre Franceschini, il Volterrano, a villa "La Petraia": iconografia medicea e orgoglio dinastico. In: Fasto di corte, a cura d M. Gregori, Firenze, vol. 2, 2006, p. 13-30.

21 BALDINUCCI, F. (1681-1728), ed. 1845-1847, V, 1847, p. 149

22 LEONCINI, G. Una "Vita di J acopo Chiavistelli pittore di figure et eccellente nell'architettura a fresco, in «Rivista d'arte», XXXVII, serie quarta, I, p. 269-346 (p. 279-280); GREGORI, M. II Seicento fiorentino, catalogo della mostra Firenze 1986-1987), 3 voll. Firenze, 1986, Biografie, p. 189.

23 Per i riferimenti bibliografici si rimanda a BASTOGI, N. Le sale affrescate da Angelo Michele Colonna e Agostino Mitelli, in Fasto di corte, a cura di Mina Gregori, vol. II, Firenze, 2006, p. 60-82 (p. 60). 
d'interni, soluzioni di grande modernità su cui si formerà J acopo Chiavistelli e i giovani della sua scuola. II Colonna ed il Mitelli propongono soluzioni tipologiche caratterizzate da una loggia sovrapposta di cui quella superiore viene risolta secondo modalità che ricordano quella dipinta nel citato salone del primo piano di palazzo Lancellotti a Roma da Agostino Tassi; questa considerazione porta a riflettere ulteriormente sugli apporti dell'ambiente romano al quadraturismo anche bolognese. La straordinaria progettazione dell'inganno, in cui realtà e finzione si mescolano enfatizzati dalla luce e dal mutare del colore in rapporto al punto di osservazione, suscita nel riguardante grande meraviglia, quello stupore che è insito nel Barocco.

I due bolognesi furono a lungo attivi anche per il principe Giovan Carlo, ${ }^{24}$ fratello di Ferdinando II, che amava la raffinatezza e la vita galante e diede grande impulso alle scienze e alle arti, nel casino degli Orti Oricellari. ${ }^{25}$ ॥I principe inoltre, in palazzo Pitti aveva il suo appartamento di circa venti stanze, che occupava tutta la parte terminale dell'ala sinistra del cortile dell'Ammannati, dal piano terreno al mezzanino sopra il primo piano ${ }^{26}$, comprendeva anche gli ambienti del quartiere che erano stati della nonna Cristina di Lorena ${ }^{27}$ e aveva accesso dal cortile e da Boboli. ${ }^{28}$

Dal 1637 Giovan Carlo diede inizio ad una serie di trasformazioni che si protrassero per oltre un ventennio, trasformand o gli ambienti a lui assegnati in Pitti in veri e propri luoghi di delizie, decorati dagli artisti più significativi del momento quali ad esempio, Angelo Michele Colonna, Agostino Mitelli, Pietro da Cortona, J acopo Chiavistelli ${ }^{29}$ che, come riferisce il Baldinucci «appartiene all'arte che fu propria»; ${ }^{30}$ gli stessi artisti, salvo il Chiavistelli, furono attivi anche nell'altra residenza del principe, il casino degli Orti Oricellari, e nei libri

24 La figura del principe, secondogenito di Cosimo II e di Maria Maddalena d'Austria, è stata oggetto di uno studio approfondito da parte di Silvia Mascalchi: MASCALCHI, S. Giovan Carlo de' Medici: an Outstanding but Neglected Collector, in Seventeenth Century Florence. «Apollo». CXX,1982, 4, p. 268-272; Idem, Anticipazioni sul mecenatismo del cardinale Giovan Carlo de' Medici e suo contributo alle collezioni degli Uffizi. In: Uffizi: quattro secoli di una Galleria, atti del convegno, Firenze 1982, p. 41-82; Idem. II 'Paradiso de' Fiori' a Palazzo Pitti, in Floralia. Florilegio dalle collezioni fiorentine del Sei-Settecento, a cura di M. Mosco, M. Rizzotto, catalogo della mostra (Firenze 10 gennaio-10 aprile 1988). Firenze, 1988, p. 135-137; cfr. anche MASCALCHI, L. La Muletta. Vicende costruttive e percorsi di vita quotidiana. In: Vivere a Pitti. Una reggia dai Medici ai Savoia a cura di S. Bertelli, R. Pasta, Firenze, 2003, p. 181-215; Idem. I Mezzanini della Muletta, Firenze, 2003. Giovan Carlo fu nominato consulente spirituale di Cristina di Svezia, da Innocenzo X.

25 II casino degli Orti Oricellari in via della Scala gli era stato assegnato dal granduca Ferdinando II il 24 agosto 1640 .

26 BALDINI, L. Ancora sul Chiavistelli: un episodio dimenticato, in L'architettura dell'inganno. Quadraturismo e grande decorazione nella pittura di età barocca, a cura di F. Farneti, D. Lenzi, atti del convegno (Rimini 28-30 novembre 2002), Firenze, 2004, p. 59-65 (p. 59).

27 Cristina era morta nel 1636.

28 Faceva parte dell'appartamento anche la piccola cappella ottagonale costruita per Cristina nel 1589 e riscoperta da Laura Baldini durante i lavori di restauro. BALDINI, L. La Grotta di Pietro da Cortona in Palazzo Pitti. In: Artifici d'acque e giardini. La cultura delle grotte e dei ninfei in Italia e in Europa, a cura di I. Lapi Ballerini, L.M. Medri, atti del convegno (Firenze 16-17 settembre 1998, Lucca 18-19 settembre). Firenze, 1999, p. 85-94.

29 BALDINI, L. Da Pitti a Boboli: I'arredo architettonico come mediazione fra intemo ed esterno, in Boboli 90 , atti del convegno. Firenze 1991, p. 393

30 BALDINUCCI, F. (1681-1728), ed. 1845-1847, IV, 1846, p. 664. Secondo Luigi Lanzi, nella prima edizione del suo testo, il Chiavistelli e Giuseppe Tonelli, gli unici quadraturisti fiorentini di cui parla, «impegnano la curiosità de' cittadini piuttosto che quella degli esteri» dando poca considerazione alla scuola fiorentina: LANZI, L. Storia pittorica dell'Italia inferiore, cioè della scuola fiorentina, senese, romana e napoletana. Firenze, 1792, p. 143. 
contabili non sempre si riesce a distinguere la destinazione dei pagamenti per i due cantieri decorativi condotti quasi contemporaneamente.

Come riferisce Lucia Mascalchi, ${ }^{31}$ le stanze terrene degli appartamenti del principe comprese fra il monumentale vestibolo di accesso allo scalone elicoidale dell'Ammannati e la testata del portico, erano occupate dalla credenza, che nel nuovo assetto venne collocata nella «camera nuova allato al portone del cortiletto», dal tinello, adiacente la cucina segreta, dalla cucina segreta, dalla bottig lieria e dagli appartamenti-atelier di significativi impiegati della corte, fra cui l'architetto Alfonso Parigi che diresse i lavori di riplasmazione. ${ }^{32} \mathrm{G}$ iovan Carlo de' Medici individuò nel mezzanino della Muletta ${ }^{33}$ la zona dove sviluppare il nuovo progetto abitativo e decorativo a partire dal 1637, e portò avanti i lavori di riplasmazione dal 1638, anno in cui al principe venne conferito l'incarico di Generalissimo dei Mari di Spagna.

Situato da un lato sotto il loggiato sinistro del cortile principale e dall'altro ad una quota inferiore del giardino, il mezzanino si sviluppava in corrispondenza dell'appartamento terreno e del primo piano di Giovan Carlo. Una piccola scala ancora esistente, posta nel piccolo andito del ninfeo, nel primo impianto del mezzanino costituiva il collegamento verticale principale fra gli ambienti dei quartieri del principe. Prima dei lavori degli anni cinquanta del Seicento, il mezzanino comprendeva un grande ambiente, la cosiddetta camera dell'Udienza; dalla documentazione archivistica si evince che questo ambiente si trovava in corrispondenza della sala terrena utilizzata da Giulio Parigi, che verrà a far parte del quartiere del principe. Adiacente alla sala maggiore si trovava una stanza più piccola a pianta quadrangolare, trasformata in una vera e propria grotta artificiale con ninfeo nella parete di fondo, ${ }^{34}$ collegata ad un vestibolo. Giovan Carlo, divenuto cardinale il 14 novembre 1644 , affidò la progettazione e la decorazione dei due ambienti del mezzanino a Pietro da Cortona, già impegnato a più riprese per il granduca nelle sale del piano nobile del palazzo.

Pietro da Cortona, che aveva già lavorato per il principe nel casino di via della Scala, ${ }^{35}$ sulla volta della grotta ninfeo dipinse il Sacrificio di Noè mentre nella sala la Nave di Argo. La presenza del cappello cardinalizio unito allo stemma mediceo nell'affresco pone il novembre 1644 come termine post

31 MASCALCHI, L. La Muletta. Vicende costruttive e percorsi di vita quotidiana. In: Vivere a Pitti, a cura di S. Bertelli, R. Pasta, Firenze 2003, p. 186.

32 Palazzo Pitti, un organismo architettonico e le schede di catalogo. In: «Bollettino d'Arte», LXIV, 1979, 1, p. 79-102 (p. 83); FACCHINETTI, F. Le vicende costruttive, in CHIARINI, M. (a cura di), Palazzo Pitti. L'arte e la storia. Firenze, 2000, p. 35.

33 II mezzanino prende nome dal bassorilievo marmoreo raffigurante una mula, murato sulla testata del portico, sotto la nicchia con Ercole in riposo.

34 I lavori furono completati entro il 1647, anno dell'allontanamento definitivo da Firenze di Pietro da Cortona. BALDIN GUSTI, L. La Grotta di Pietro da Cortona. In: Palazzo Pitti... cit., p. 85-94.

35 II pittore e architetto cortonese aveva dipinto sul soffitto della camera da letto terrena del principe l'Allegoria della Quiete, fra la fine del 1642 e l'inizio del 1643. MEGALE, T. Precisazioni intomo all'«Allegoria della Quiete »di Pietro da Cortona. In: «Paragone-Arte», XL, 1989, 17, p. 89-93. 
quem della progettazione e dell'inizio dei lavori che termineranno entro il 1647, anno dell'allontanamento definitivo di Pietro da Cortona da Firenze. Negli stessi anni Quaranta fu attivo per Giovan Carlo anche Salvator Rosa, pittore amatissimo dal cardinale, ${ }^{36}$ che intorno al 1645 realizzò le quattro lunette della sala maggiore con episodi biblici legati all'elemento dell'acqua: il Ritrovamento di Mosè, Mosè e il roveto ardente, Agar e Ismaele, Tobiolo e l'angelo. ${ }^{37}$ Q uesti «quattro quadri» costituiscono l'unico intervento fino ad ora conosciuto di Salvator Rosa come frescante. L'arredo pittorico della grotta e della sala adiacente può ritenersi concluso nel 1647, quello plastico a stucco e a scagliola delle pareti, caratterizzate da un rivestimento polimaterico i cui cromatismi alludono all'acqua come il tema degli affreschi, venne condotto a partire dal 1656 su progetto di Pietro da Cortona; fra i manifattori emerge il romano Silvio Alli, maestro specializzato in apparati decorativi in stucco e scagliola. ${ }^{38}$ Per il principe cardinale il plasticatore decorò anche il «salottino detto il Paradiso de' Fiori» nel corrispondente appartamento del primo piano, un altro ambiente esclusivo in cui intervenne anche J acopo Chiavistelli.

Nel 1659 il principe card inale fece eseguire lavori «nello stanzone terreno», 39 presumibilmente quello corrispondente al mezzanino, che potrebbero essere intesi in rapporto alla costruzione del solaio che portò all'ampliamento del mezzanino con una sala ex novo molto ampia. II nuovo spazio metteva in comunicazione il mezzanino del principe con quello situato nella stessa ala del palazzo destinato al cardinale Leopoldo, tramite un accesso interno più dignitoso, che si apriva sulla prima rampa della scala a «umaca» dell'Ammannati, ${ }^{40}$ collegamento verticale principale fra gli ambienti dei contigui appartamenti e di Giovan Carlo.

Si presume che in seguito a questi lavori, siano intervenuti J acopo Chiavistelli e Andrea Ciseri non per dipingere la volta della nuova sala, come si evince da un documento d'archivio che riferisce «nel Mezzanino grande della Fonte fatto fabbricare e dipingere tutto fino in terra dal lombardo Chiavistelli» ${ }^{41}$, ma per realizzare la decorazione delle pareti adattandole alle nuove esigenze visive.

36 CHIARINI, M. e NOEHLES, K. Pietro da Cortona a palazzo Pitti. Un episodio ritrovato. In: «Bollettino d'arte», LII, 1967, p. 233-239; CHIARINI, M. L'appartamento d'Inverno. In: Palazzo Pitti... cit., p. 117.

37 BALDINI GIUSTI, L. La grotta di Pietro da Cortona in Palazzo Pitti... cit., p. 85-94. La piccola stanza ad uso bagno, con vasca a contorno mistilineo, si trovava adiacente la sala decorata da Pietro da Cortona e Salvator Rosa. L'ambiente, andato perduto nel 1815 durante i lavori di modifica della piccola scala di servizio, è stato ricomposto nei recenti restauri del mezzanino diretti da Laura Baldini.

38 Per approfondimenti su Silvio Alli si rimanda a MASCALCHI, S. Il ‘Paradiso de’ Fiori’ a Palazzo Pitti... cit., p. 136 nota 21.

39 FIRENZE, Archivio di Stato (=ASF), Scrittoio delle Regie Possessioni. 4257, c. XIIII, nota di spesa del 30 giugno 1659 e c. LXX.

40 La scala è andata distrutta negli interventi ottocenteschi realizzati dal Poccianti, per l'apertura del «passare» di comunicazione fra il cortile dell'Ammannati e il cortile delle Colonne e la successiva edificazione dello scalone.

41 ASF, Misc. Med. 312, ins. 10, Inventario di tutte le robe che si ritrovano nella Guardaroba del ser.mo principe Cardinale Gio. Carlo di felice memoria nel palazzo de Pitti nelle stanze di sua abitazione e fonderia sotto la chiave e custodia di Francesco Conti. 9 maggio 1663, c. 74r 
Dalle note di spesa si evince una intensa attività dei due artisti per Giovan Carlo negli ultimi anni Cinquanta; purtroppo gli apparati pittorici realizzati nelle sale dell'appartamento del principe cardinale sono andati perduti. Quasi contemporaneamente i due maestri fiorentini dipingevano gli ambienti dell'appartamento d'estate del principe Cosimo e della sua sposa Marguérite Louise d'Orléans, a terreno nell'ala destra del cortile, in collaborazione con Francesco Betti e Cosimo Ulivelli per le figure, Agnolo Gori per i festoni di frutta e fiori.

Se l'apertura ottocentesca del corridoio di comunicazione fra il cortile dell'Ammannati e quello delle Colonne portò alla demolizione della scala a lumaca, la costruzione del nuovo scalone Poccianti, realizzato nell'Ottocento, interessò la sala terrena e l'ultima stanza annessa al mezzanino di Giovan Carlo, il cui apparato decorativo venne così tag liato in due parti da un muro. La porzione più consistente della volta è ancora visibile nell'ultimo vano che fa parte dei mezzanini del Museo degli Argenti, con la specchiatura centrale dominata dalla figura allegorica della Fama che regge un nastro con il motto «Al suo nome la terra e 'I mar rimbomba», riferita alla nomina di Giovan Carlo a Generalissimo del mare nel 1638; nell'altro, due putti alati reggistemma. (fig. 1)

Nell'apparato pittorico dei due ambienti che si sono così costituiti, sono riconoscibili due mani distinte, una responsabile della volta, l'altra delle pareti. La macchina architettonica dipinta sulla copertura attorno all'allegoria della Fama, come ha dimostrato lo studio geometrico, evidenzia un impianto prospettico studiato e realizzato per una visione a distanza, dal salone terreno e non da un punto di vista ravvicinato; inoltre, la parte superiore dei peducci in pietra su cui si imposta la volta, a circa un metro da terra, è lasciata a grezzo. Queste considerazioni porterebbero a datare l'apparato decorativo ad un periodo precedente ai lavori di ampliamento del mezzanino. II repertorio architettonico illusionistico così ricco di elementi decorativi e figurativi, mostra evidenti analogie con gli apparati dipinti dal Mitelli e dal Colonna, pittori legati a Giovan Carlo da significative commissioni e così importanti per la formazione di] acopo Chiavistelli, analogie che interessano: l'organizzazione dello spazio di copertura mediante diversi livelli prospettici giocati su elementi curvilinei e poligonali; la tipologia delle piccole balaustre analoghe, ad esempio, a quelle del salone di Sassuolo; il repertorio figurativo con ninfe per le quali possiamo ricordare la sala di villa Arnolfini a Gragnano; i fauni; i medaglioni a fingere bassorilievi, cinti da cornici dorate; ${ }^{42}$ la morfologia della valva di conchiglia, motivo ampiamente documentato anche nei cantieri fiorentini condotti dai due maestri bolognesi non solo nelle proprietà

42 L'inserimento di medaglioni nel costrutto architettonico virtuale viene documentato anche nei numerosi disegni che ci sono pervenuti, ad esempio il n. 1347 della Kunstbibliothek di Berlino oppure la decorazione della cappella del Rosario in San Domenico a Bologna. 
medicee ma anche nella galleria di palazzo Niccolini, andata perduta ma documentata dai disegni di G iuseppe Menabuoi, ${ }^{43}$ in cui il Colonna fu attivo con Giacomo Alboresi. Queste considerazioni attributive trovano conferma nella biografia scritta da Giovanni Mitelli, figlio di Agostino, in cui afferma che Giovan Carlo era «invaghito della sua [... ] et singolare Maniera» e che il padre mentre dipingeva nel casino di via della Scala, decorò «una stanza bassa a Palazzo pel med(esi)mo S(igno)r Card(ina)le Gio. Carlo» assieme al Colonna, presumibilmente da identificare con quella stanza che venne in seguito soppalcata e annessa al mezzanino ${ }^{44} \|$ riferimento al «ombardo Chiavistelli» contenuto nell'Inventario, ${ }^{45}$ deve quindi essere riferito alle lunette della sala nelle quali, diversamente dall'apparato ornamentale della volta, la prospettiva sembra impostata per una visione dal piano del mezzanino. II confronto stilistico dei decori eseguiti dal maestro fiorentino, e non lombardo come riferisce il Marmi, mette in evidenza una notevole vicinanza con il repertorio dei due pittori bolognesi e gli apparati architettonici virtuali da loro realizzati. Del resto sappiamo quanto il Chiavistelli abbia studiato e copiato le realizzazioni del Colonna e del Mitelli, quindi perfettamente in grado di dare una giusta interpretazione all'ingannevole costrutto del mezzanino realizzato dai bolognesi. Inoltre il Colonna conosceva molto bene Andrea Ciseri che era stato scelto da lui tra i giovani che sapevano «tirar due linee e dare le prime tinte» ${ }^{46}$ mentre decorava le stanze del quartiere terreno di Ferdinando II in palazzo Pitti; un rapporto che continua nel cantiere decorativo di palazzo Niccolini. Si può presumere infatti che sia stato proprio il bolognese a segnalare nel 1652 il giovane pittore, che aveva da poco contratto un sodalizio con J acopo Chiavistelli, al marchese Filippo Niccolini ${ }^{47}$ per la decorazione di alcuni ambienti del palazzo di via dei Servi a Firenze, mentre lui stesso vi stava lavorando assieme al Mitelli.

II Chiavistelli fu attivo in palazzo Pitti fin dagli anni Cinquanta, come attesta una nota di pagamento in data 1 marzo 1655 riferita a «più pitture da lor (Chiavistelli e Ciseri) fatte alla capannuccia del Ser.mo Principe Francesco». Nei primi anni Settanta, con la ridefinizione degli spazi della residenza granducale dopo la morte di Ferdinando II sopraggiunta il 27 maggio 1670, la granduchessa madre Vittoria della Rovere commissionò al prospettico la

43 Si rimanda a FARNETI, F. Tra realtà e illusione: le architetture dipinte nei palazzi fiorentini, in BEVILACQUA, M., MADONNA, M.L. (a cura di), Atlante tematico del Barocco in Italia. Residenze nobiliari. Stato pontific io e Granducato di Toscana, Roma 2003, p. 327-348 (p. 328-329).

44 BOLOGNA, Biblioteca Comunale Archiginnasio, ms. 3375, Vita et Opere di Agostino Mitelli pittore bolognese descritte dal figlio Giovanni. I documenti d'archivio registrano la presenza a Firenze del Colonna negli anni 1644, 1648-52, 1657. A Firenze il pittore godeva di una tale stima che il principe Leopoldo de' Medici desiderava avere il suo ritratto da esporre nell'Accademia del Disegno.

45 ASF. Miscellanea Medicea. f. 312, c. 74r. L'Inventario fu compilato dopo la morte del cardinale

46 FIRENZE, Biblioteca Mediceo Laurenziana, ms. Antinori 248, Vite e Notizie di uomini chiari, c. 5v; il manoscritto è stato pubblicato da LEONCINI, G. Una "Vita di J acopo Chiavistelli pittore di figure et eccellente nell'architettura a fresco". In: «Rivista d'Arte», XXXVII, 1984, 1, p. 269-346

47 Filippo Niccolini ricopriva importanti uffici a corte 
decorazione della stanza da letto nel suo quartiere terreno, ${ }^{48}$ posto nell'ala sinistra del cortile dell'Ammannati, attiguo a quello del marito Ferdinando, detto attualmente 'degli Argenti' e, qualche anno più tardi, i decori di altri ambienti e della cappellina in cui dipinse «intorno al basso rilievo». Di questo ampio intervento sono sopravvissuti integri gli ingannevoli apparati architettonici della stanza di Vittoria, affacciata sul cortile ammannatesco, della stretta galleria di raccordo con un «ricetto»e, con gravi lacune, la macchina architettonica dipinta da J acopo Chiavistelli nella sala prospettante il cortile Non Finito che rimase tagliata in due quando il Poccianti costruì, nel 1815, il 'nuovo passare' di collegamento fra i due cortili. La camera da letto di Vittoria, già appartenuta a Maria Maddalena d'Austria, mostra uno schema decorativo codificato dalla tradizione, dove il portico scenografico costituisce uno dei motivi più peculiari, l'elemento cardine del costrutto architettonico illusionistico, un tema che verrà ampiamente riproposto a Firenze fino al Settecento inoltrato in numerose varianti, ma sempre essenziali nell'intento di dilatare, diaframmare e, talora, dare nobiltà ad ambienti altrimenti disadomi. Marco Chiarini ha identificato in un disegno conservato al Gabinetto Disegni e Stampe degli Uffizi, ${ }^{49}$ uno studio progettuale per la decorazione di questo ambiente caratterizzata da un monumentale portico dai colori chiari dell'architettura reale, impostato su un basamento arricchito da festoni di frutta e mascheroni cordonati, propri del linguaggio chiavistelliano, che si apre su quinte sceniche di cortili. La soluzione d'angolo tra pareti e copertura voltata, dove è raffigurata una Allegoria del Sonno, attuata mediante archi trasversali, troverà una sua evoluzione nei costrutti dei suoi principali allievi, quale ad esempio Rinaldo Botti.

Dieci anni prima il Chiavistelli ed il C iseri, con l'aiuto di Francesco Bettini, Cosimo Ulivelli e Agnolo Gori, erano stati attivi per il gran principe Cosimo, che salirà agli onori granducali nel 1670 alla morte del padre Ferdinando II. Infatti nel 1661, in occasione del suo matrimonio con la giovane Marghérite-Louise d'Orléans, cugina del re di Francia Luigi XIV, Cosimo diede inizio a lavori di riammodernamento del palazzo e si rivolse al prospettico per la decorazione di nove ambienti del suo quartiere terreno e di quello della moglie, nell'ala destra del cortile ammannatiano, «un salone e otto camere, tutte dipinte a fresco fino al pavimento, e veramente cercò (il Chiavistelli) di sforzarsi quanto poté nei pensieri e nel colorito d'avvicinarsi alla maniera del Colonna ${ }^{50}$. L'appartamento ha subito successive manomissioni a scapito delle decorazioni parietali; il grande salone centrale nel 1766, per volere di Pietro Leopoldo, fu trasformato in cappella Palatina e la maggior parte delle

48 II quartiere era stato in uso al cardinale Leopoldo.

49 FIRENZE, Gabinetto Disegni e Stampe Uffizi,, n. 5264 A, foglio n. 181. Seppia, acquerelli colorati 290x 445mm. II disegno reca in alto la scritta «Di J acopo Chiavistelli fece à Pitti nelle stanza terrene dove abitava la GD.a Vitt.a».

50 LEONCINI, G. Una "Vita di J acopo Chiavistelli pittore di figure et eccellente nell'architettura a fresco". In: «Rivista d'Arte», XXXVII, 1984, 1, p. 269-346 (p. 326). 
stanze scialbate nel XIX secolo. ${ }^{51}$ Bocchi e Cinelli hanno descritto con grande precisione l'apparecchiatura architettonica virtuale delle pareti del salone «detto di Apollo» percorse da un finto loggiato che lasciava vedere «fabbriche nobili»; la volta si apriva con tre sfondi, quello centrale con l'arme del principe e gli altri due con le figure di Diana e Apollo sul carro, delimitati da un finta architettura di stucchi bianche e dorati. ${ }^{52}$ Solo due sale mantengono un apparato pittorico abbastanza integro che mostra il lessico chiavistelliano con una partitura parietale realizzata da pilastri, nicchie, balaustrate oltre le quali, talora, si aprono unghie acute enfatizzate dalle figure di nereidi che troveranno un seguito negli apparati dell'inganno realizzati dal Botti. Nella decorazione dei soffitti delle sale la fantasia tardo-manieristica del Chiavistelli si coniuga con l'insegnamento dei bolognesi, realizzando effetti sorprendenti e straordinariamente decorativi, con abili sotto in su. (fig. 2)

Nel 1666 il Chiavistelli fu impegnato negli ornati della biblioteca palatina al secondo piano del palazzo, dipinse un fregio che percorreva le pareti al di sopra degli scaffali, andata perduta durante i lavori ottocenteschi, ma di cui si conservano testimonianze grafiche..$^{53}$

In occasione delle nozze del gran principe Ferdinando, figlio di Cosimo III, con la principessa Violante di Baviera, celebrate nel gennaio del 1689, anche palazzo Pitti fu oggetto di lavori radicali, strutturali e decorativi, che riguardarono l'appartamento di Ferdinando collocato nel piano nobile prospettante piazza Pitti, ${ }^{54}$ quello retrostante detto «dei principi e forestieri», assegnato alla principessa, e i mezzanini sempre del gran principe, ambienti che sono stati in gran parte interessati dalle ristrutturazioni lorenesi e sabaude. Fu Francesco Sacconi, allievo del Chiavistelli, ${ }^{55}$ nel 1686 a realizzare assieme a Pier Dandini e a Bartolomeo Bimbi la decorazione del piccolo «gabinetto» segreto di Ferdinando, progettato da Giacinto Maria Marmi, un arredo pittorico a monocromo, dal bianco al grigio, che riprendeva alcuni motivi impiegati nella parte lignea ad intaglio dell'ambiente, vivacizzato dalla cromia dei tralci di fiori dipinti dal Bimbi, dalla profusione dell'oro giallo e brunito, del tenue azzurro che cambia in violetto nei pannelli della volta e nei medaglioni del Dandini. Nell'appartamento di Violante si rileva la decorazione della piccola cappella dedicata alla Vergine, mal conservata e ridotta a toilette, che nell'apparecchiatura ad illusionismo architettonico e nel repertorio decorativo della parte alta costituito da festoni, putti, serti,

51 Il descialbo condotte in alcune stanze ha riportato alla luce brani di architettura virtuale.

52 FIRENZE, Biblioteca Nazionale Centrale, ms. 20-6-239, F. Bocchi, G. Cinelli.

53 FACCHIANETTI, F. La Biblioteca Palatina. In: Palazzo Pitti L'arte e la storia, a cura d M. Chiarini, Firenze 2000, p. 87-89; BAROCCHI, P. e GAETA BERTELÁ, G. Arredi principeschi dei Seicento fiorentino. Torino 1990, p. 84-85.

54 Una descrizione dell'appartamento del principe Ferdinando, che era stato parzialmente abitato dal cardinale Carlo, fratello di Cosimo II, fino alla morte nel 1661, in ASF, Guardaroba, 1222; Guardaroba, 1067,anno 1698.

55 Per la sua biografia si rimanda a FARNETI, F. In: FARNETI, F. BERTOCCI, S. L'architettura dell'inganno a Firenze. Firenze, 2002, ad vocem, p. 83-88. 
volute mostra la mano del Chiavistelli che era entrato stabilmente al servizio di Ferdinando dal 1688.

La maggior parte degli ambienti dei mezzanini di Ferdinando, situati nell'ala destra del palazzo prospettante la facciata di Pitti, costituiti da almeno venti ambienti in gran parte trasformati nel corso del Settecento e dell'Ottocento, furono decorati ad illusionismo architettonico; la documentazione archivistica infatti ricorda la presenza di artisti, quasi tutti quadraturisti, quali Luca Bocci, Ercole Graziani, J acopo Chiavistelli. Dell'appartamento interamente decorato e stuccato, molto apprezzato dai contemporanei e, nel Settecento, da Montesquieu e da Charles de Brosses, sopravvivono con la decorazione originaria la Meridiana e la contigua galleria, in cui il Chiavistelli intervenne con il Gabbiani fra il 1693 ed il 1694. Il soffitto voltato di quest'ultima viene tripartito mediante plastiche cornici architettoniche che costruiscono il primo livello prospettico, su cui si impostano arcate trasversali, che suggeriscono la presenza di illusionistici ambienti superiori, in analogia con soluzioni decorative degli ambienti inferiori del quartiere di Cosimo. Le quinte prospettiche che dilatano lo spazio reale, sono evidenziate dalla diversa cromia. (fig. 3 )

II rinnovamento di palazzo Pitti portato avanti Ferdinando trova il suo culmine nell'affrescatura delle sale del quartiere estivo a terreno ubicato nella parte destra dell'edificio, di cui fra il 1706 ed il 1707 Giuseppe Tonelli, grande protetto del gran principe e attivo per lui dal 1688 come allievo del Chiavistelli, e Sebastiano Ricci affrescarono l'anticamera di modeste dimensioni. Sulle pareti lunghe della stanza il prospettico costrui un portico ionico, coperto a cassettoni, di chiavistelliana memoria, su cui si imposta un piano balaustrato. La macchina dell'inganno, costruita con grande credibilità strutturale esibendo gli spessori dei vari elementi, si apre sulla volta ad esaltare la specchiatura figurativa con Venere ed Adone, dipinta dal Ricci.(fig. 4 ) Sulla parete di fondo il figurista bellunese ricorre ad una soluzione sorprendente; dipinge, infatti, lo stemma mediceo, di grande evidenza plastica, sormontato dalla corona granducale e sorretto da due elegantissimi angeli.

L'angelo di destra manifesta la quint'essenza dello stile del Ricci, intesa in termini di eleganza formale e libertà inventiva delle pose: volge lo sguardo nella posizione opposta all'osservatore, in un movimento di torsione all'indietro che implica la flessione della gamba sinistra di cui si riesce a intravedere solo la coscia, l'altra invece è protesa verso il il riguardante a mostrare tutta la sua agile lunghezza. A questa figura sembra ispirarsi Lorenzo del Moro per la figura a monocromo dipinta sopra la finestra del lato destro della navata dei Santi Filippo e Prospero a Pistoia.

Con i lavori commissionati dal gran principe Ferdinando si chiude in palazzo Pitti la grande stagione del quadraturismo barocco fiorentino. I successivi interventi di riammodernamento decorativo, sette e ottocenteschi, condotti da artisti quali ad esempio, Giuseppe Castagnoli, Luigi Catani e Luigi 
Ademollo mostrano apparati architettonici con limitati effetti di illusionismo spaziale, un ornato che simula sempre più lo stucco, con una predilezione per le monocromie.

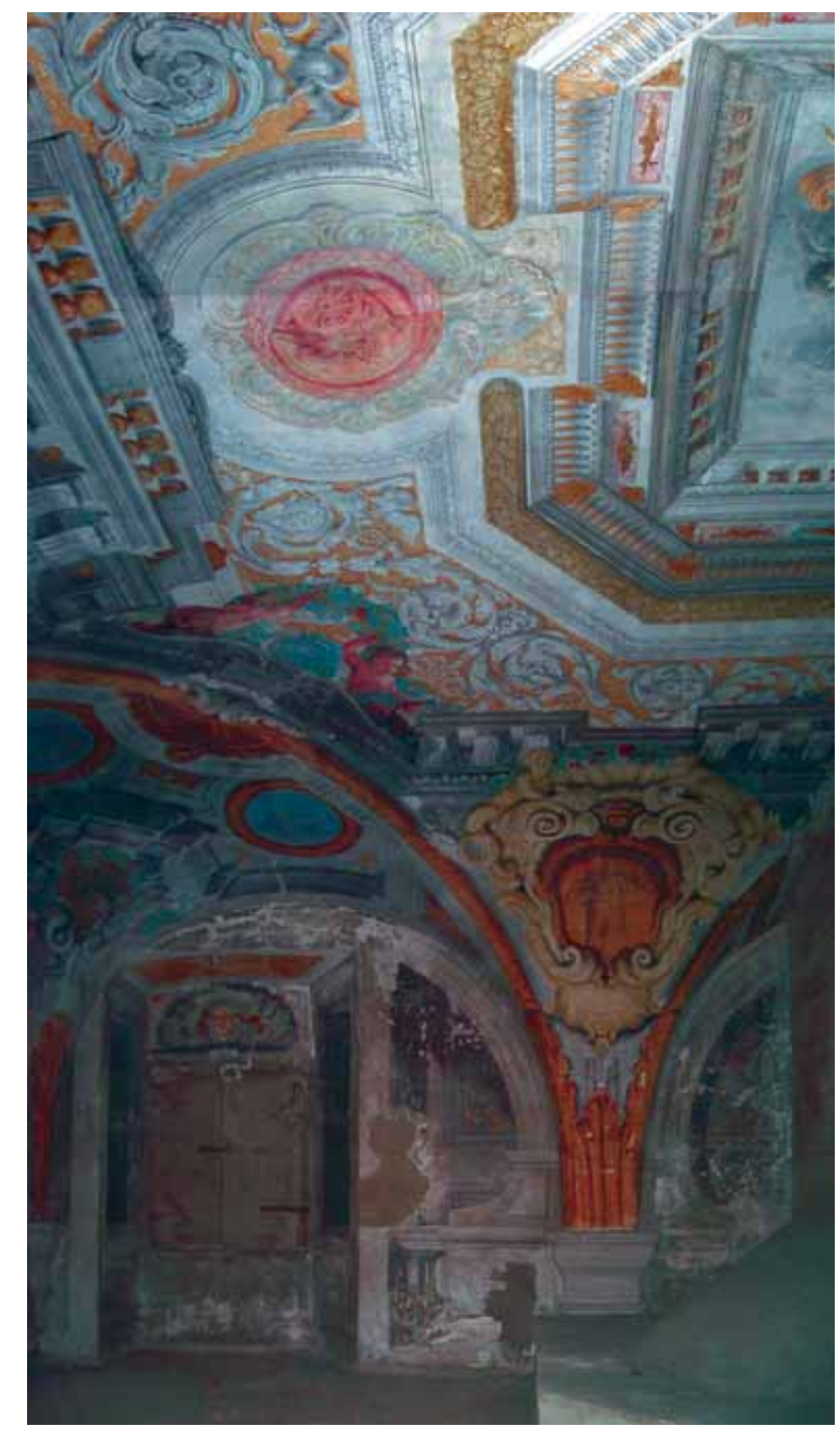

Figura 1: Palazzo Pitti, mezzanino della Muletta, decorazione ad illusionismi architettonici. 


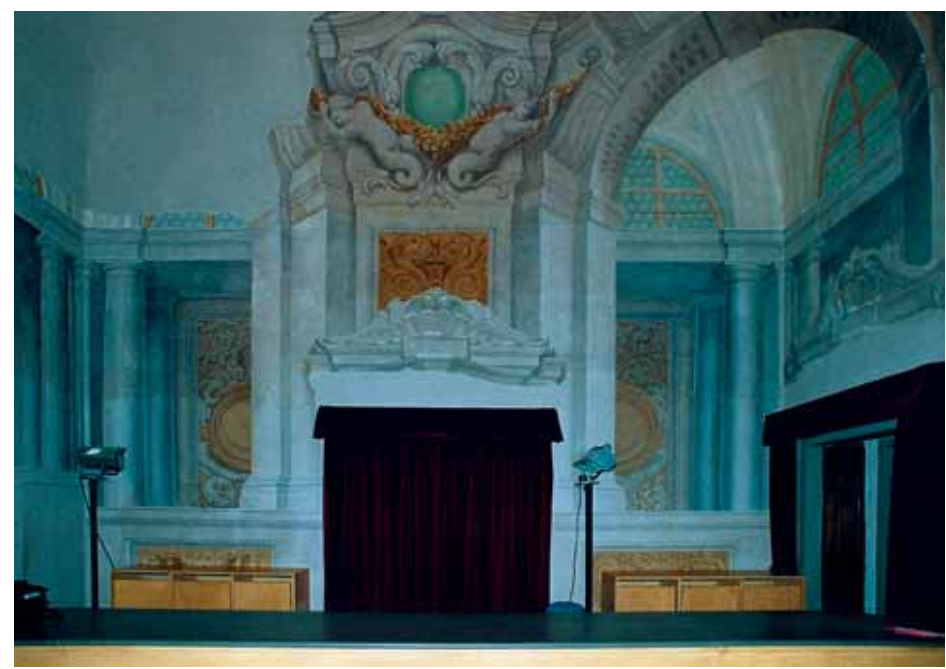

Figura 2: Palazzo Pitti, quartiere terreno di Cosimo III, J acopo Chiavistelli.

Figura 3: - Palazzo Pitti, mezzanino del gran principe Ferdinando, decorazione della volta della galleria, J acopo Chiavistelli, Anton Domenico Gabbiani.

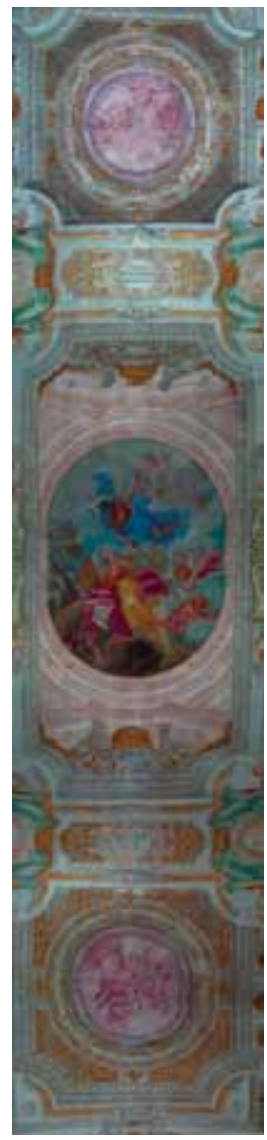




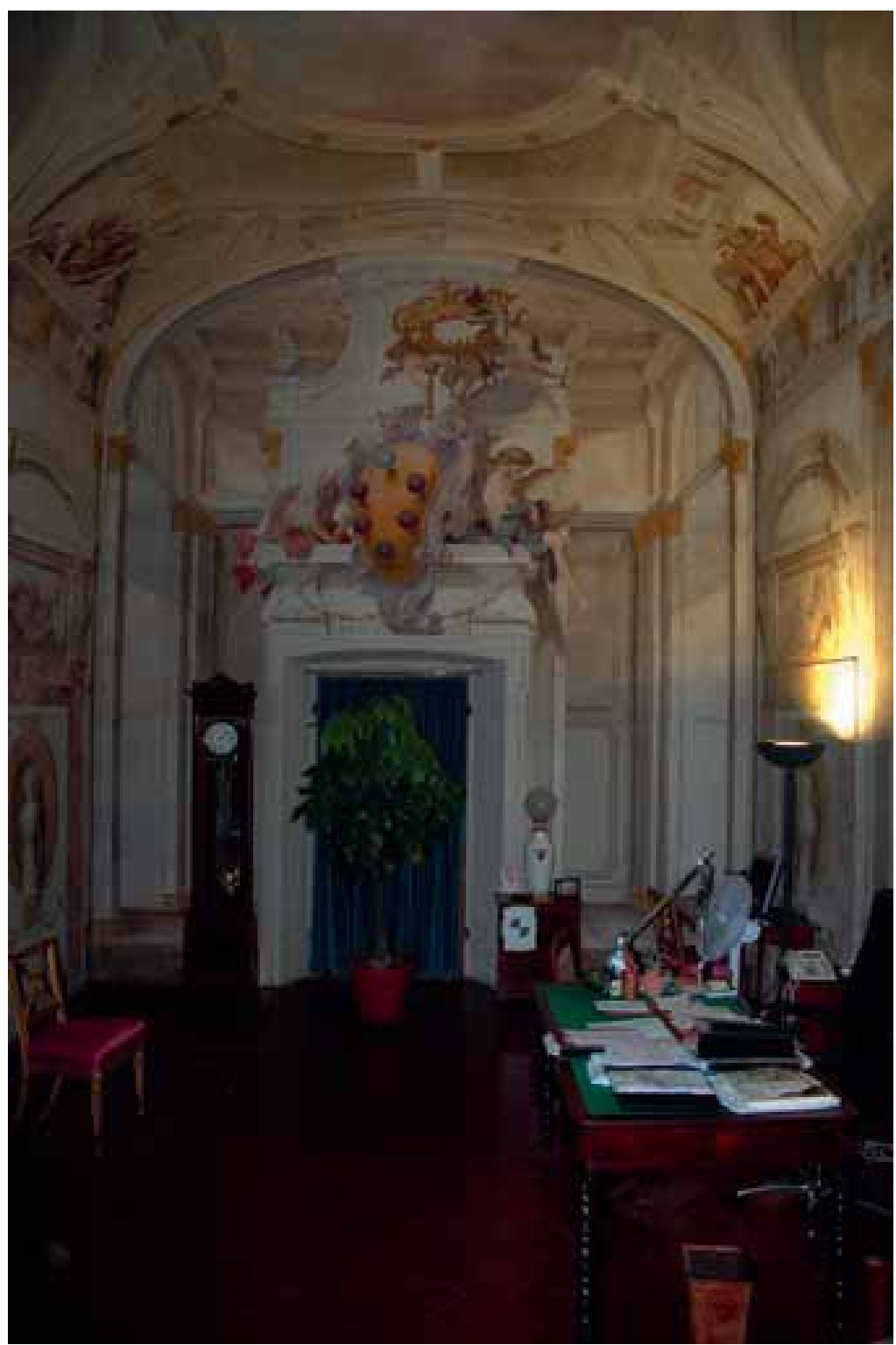

Figura 4: Palazzo Pitti, anticamera del quartiere estivo del gran principe Ferdinando, Giuseppe Tonelli, Sebastiano Ricci. 